\title{
Research on the Characteristics of the Low Voltage Power Line Carrier Communication Channel
}

\author{
Bochuan Wang, Xixiu Wu, Zhuqing Xia, Xin Yang, and Zhining Yang
}

\begin{abstract}
Low voltage power line is widely used for carrier communication, whose channel characteristics is an important factor to the quality of carrier communication. In order to make a model analysist to obtain the input impedance characteristics, attenuation characteristics and noise characteristics of low voltage power line carrier communication. This paper put forward a model for the communication channel by analyzing the characteristics of low voltage power line communication channel. The simulation result could accurately reflect the characteristics of channel noise, which matches the data measured in the laboratory environment very well. The spectrum analysis of measured noise data and the simulation result of power line impedance and attenuation characteristics show that the noise, impedance and attenuation between $120 \mathrm{kHz} \pm 20 \mathrm{kHz}$ band is relatively small, so the band is suitable for low voltage power line carrier communication.
\end{abstract}

Index Terms-Low voltage power line, carrier communication, impedance characteristics, attenuation characteristics, noise characteristics.

\section{INTRODUCTION}

PLC(Power Line Communication)is a way of communication using power lines as a communication medium. Due to the numerous power line nodes and the existence of various capacitive and inductive loads, the power line is not an ideal communication medium. However, with the continuous development of the modulation and demodulation technology and the microelectronic technology, the widespread use of PLC has gradually become possible.

When the low voltage power line is used as a high frequency carrier channel, it must overcome the complex electrical characteristics of the power line channel, which is completely different from the dedicated communication channel, mainly includes three aspects: (1) Change regularities of input impedance of carrier signal access point (2) Transmission attenuation characteristics due to reflection and refraction caused by impedance mismatch nodes in the channel. (3) The random cut in, cut out of the power line load and various switch on and off movement, which lead to the noise interference characteristics [1].

The above three characteristics are the main factors affecting the reliability of power line communication. This paper establishes the simulation models by analyzing three characteristics of power line, and comparing the simulation

Manuscript received October 30, 2018; revised December 18, 2018.

Bochuan Wang, Xixiu Wu, Zhuqing Xia, Xin Yang, and Zhining Yang are with WuHan University of Technology, Wu Han, China (e-mail: wbc_king@163.com, wuxixiu@163.com, Content_Xia@163.com, yangxin_0614@163.com,1057248213@qq.com). results with the measured data, then adjusting the model parameters [2]. Which finds that the simulation results can response to the change law of the measured data very well, so the model has some practicality.

\section{LOW Voltage POWER Line INPUT IMPEDENCE CHARACTERISTICS}

The power line input impedance characteristic refers to the equivalent impedance of the power line represented by the carrier signal access point [3].

As shown in Fig. 1, suppose $u$ is the signal source voltage, $r$ is the signal source internal resistance, and $R$ is the load input impedance. The power on $R$ is as shown in (1).

$$
P=\frac{\left(\frac{R u}{R+r}\right)^{2}}{R}=\frac{u^{2}}{R+\frac{r^{2}}{R}+2 r}
$$

From (1), it is known that power $p$ takes the maximum value when $R=r$. Therefore, the matching relationship directly affects the anti-attenuation capability and transmission distance of the carrier signal.

Ideally, the power line can be equivalent to a transmission line with uniformly distributed characteristics, and its input impedance should decrease as the frequency increases [4]. The change of the input impedance does not necessarily conform to this change rule when the actual load is applied. And the cut in and cut out of various complex loads make the impedance change complicated. The input impedance of the power line rang of $2 \Omega$ to $40 \Omega$ [5-6]. The impedance characteristics of different test points are very different, even for the same test point, the test results are very different in different time periods. Therefore, it is difficult to achieve complete impedance matching [7]. However, it is possible to find the signal frequency that is most suitable for carrier communication through simulation and measured data analysis. The impedance and attenuation characteristics of the channel will increase significantly when the signal frequency exceeds $150 \mathrm{kHz}$.

\section{LOW Voltage POWER Line AtTENUATION CHARACTERISTICS}

Carrier signal attenuation is divided into two stages: first, coupling attenuation due to impedance mismatch; second, line loss, multipath transmission attenuation and delay attenuation, collectively referred to as line attenuation [8].

The power line network has many branches and nodes, that are so many discontinuous points, which cause the 
signal to reflect at the node, so that the signal cannot be directly transmitted to the receiving end, so the signal power is greatly attenuated, which seriously affects the communication distance.

$$
H(f)=\sum_{i=1}^{n} a_{i} \cdot E\left(f, d_{i}\right) \cdot e^{-j \cdot 2 \pi f \tau_{i}}
$$

Equation (2) is a transfer function equation of power line multipath transmission characteristics, $a_{i}$ is a weighting factor of path $i ; E\left(f, d_{i}\right)$ is an attenuation function, and its characteristics are related to parameters such as transmission distance and signal frequency; $\tau_{i}$ represents a delay of path $i$. By selecting the fixed frequency carrier signal to measure the attenuation of the actual power line grids, analyzing the attenuation measurement results of a small number of decentralized frequency points, the approximate value of the parameter in (2) can be determined, and then the carrier signal of the power line grids can be simulated with the frequency attenuation changes.

The attenuation is small but unstable when the frequency is less than $100 \mathrm{kHz}$; it is stable in the frequency range of $100 \mathrm{kHz}$ to $400 \mathrm{kHz}$; the attenuation is significantly increased when the frequency is more than $500 \mathrm{kHz}$.

\section{LOW Voltage POWER Line NoISE CHARACTERISTICS}

The power line noise is measured using the measurement circuit shown in Fig. 2.

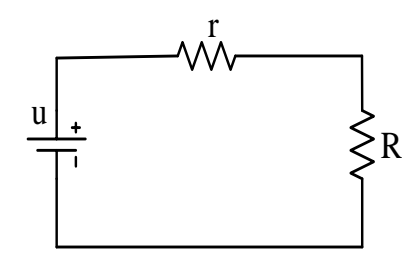

Fig. 1. Analysis of coupling principle.

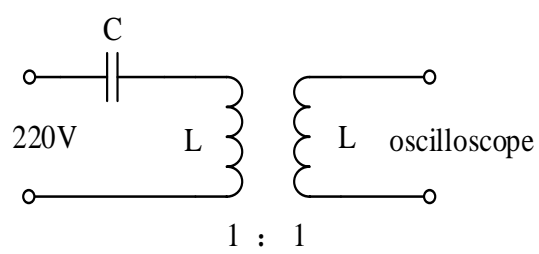

Fig. 2. Noise measuring circuit.

As shown in Fig. 2, the left side is connected to the 200V power line, and the right side is connected to the high-frequency oscilloscope or spectrum analyzer. The capacitance of the capacitor $\mathrm{C}$ is $220 \mathrm{nF}$, and the inductance of the coupler coil is $12.5 \mathrm{mH}$, so the cut off frequency is about $30 \mathrm{kHz}$.

Fig. 3 shows the periodic impulse noise waveform. Fig. 4 shows the sudden impulse noise. Impulse noise has a large amplitude and it is the main interference affecting power line communication.

Fig. 5 shows the background noise waveform. There is no specific change in background noise, and the change is faster and the amplitude is relatively stable.

Fig. 6 shows the noise spectrum from 0 to $10 \mathrm{MHz}$. The noise amplitude tends to be stable after $2 \mathrm{MHz}$, about $-60 \mathrm{~dB}$, which is much smaller than the interference amplitude in the $100 \mathrm{kHz}$ frequency range.

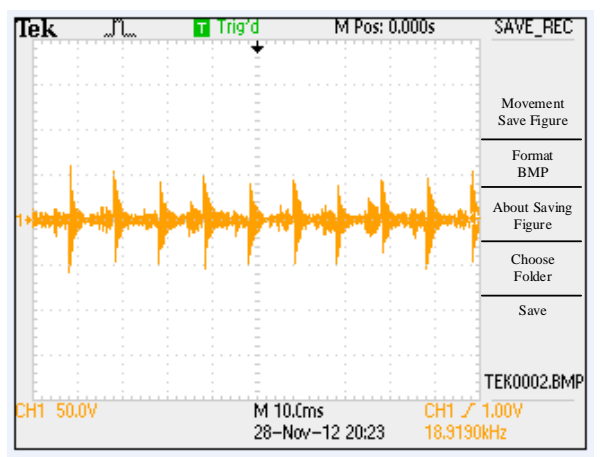

Fig. 3. Periodic impulse noise.

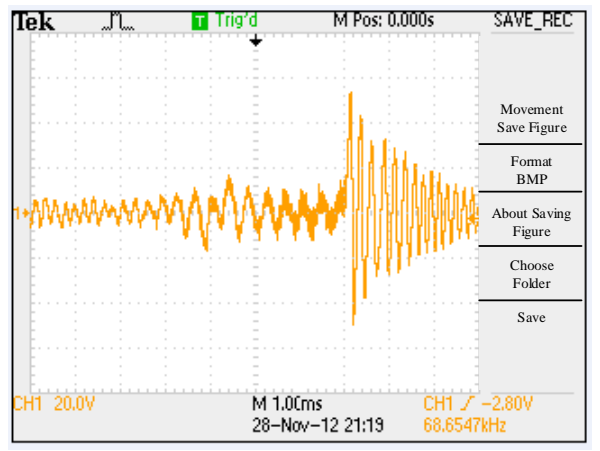

Fig. 4. Sudden impulse noise.

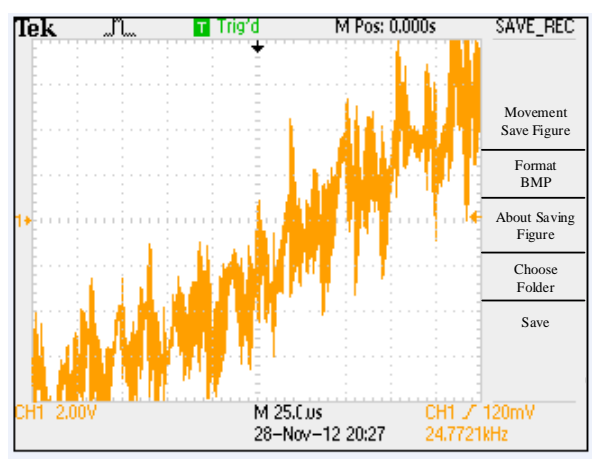

Fig. 5. Background noise.

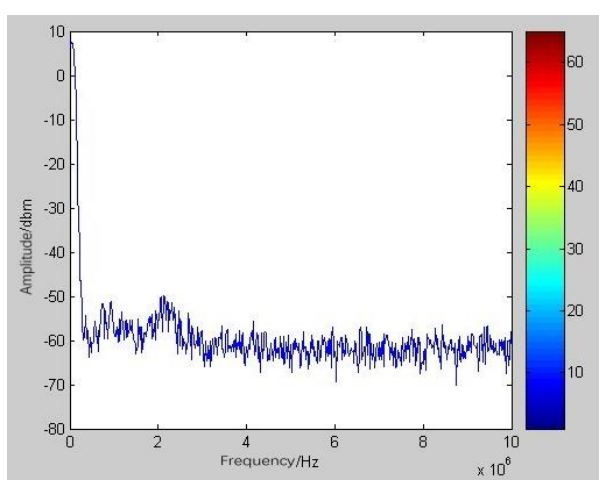

Fig. 6. Noise spectrum(0 to $10 \mathrm{MHz})$.

The measurement results of power line noise in the laboratory environment as shown in Fig. 7 and Fig. 8 show that the power line interference mainly exists in the frequency range within $100 \mathrm{kHz}$, and the interference amplitude in the frequency range more than $100 \mathrm{kHz}$ is basically stable and relatively small, about $-60 \mathrm{~dB}$.

Noise characteristics can be subdivided into five categories: (1) Colored background noise; (2) Narrowband 
noise; (3) Periodic noise asynchronous to power frequency; (4) Period noise synchronized with power frequency; (5) Sudden impulse noise.

Narrowband noise can be described as a superimposed of several independent sinusoidal functions, as following equation:

$$
N_{\text {narrow }}(t)=\sum_{i=1}^{n} A_{i}(t) \sin \left(2 \pi f_{i} t+\phi_{i}\right)
$$

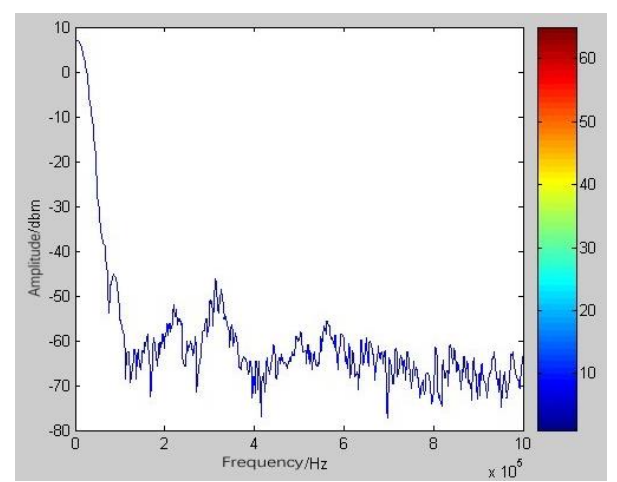

Fig. 7. Noise spectrum( $(0$ to $1 \mathrm{MHz})$.

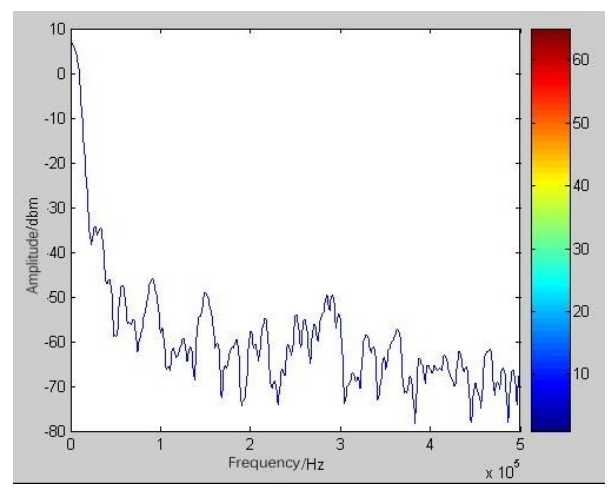

Fig. 8. Noise spectrum( $(0$ to $500 \mathrm{kHz})$.

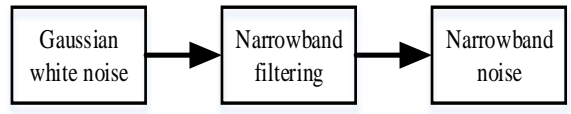

Fig. 9. Narrowband noise generation.

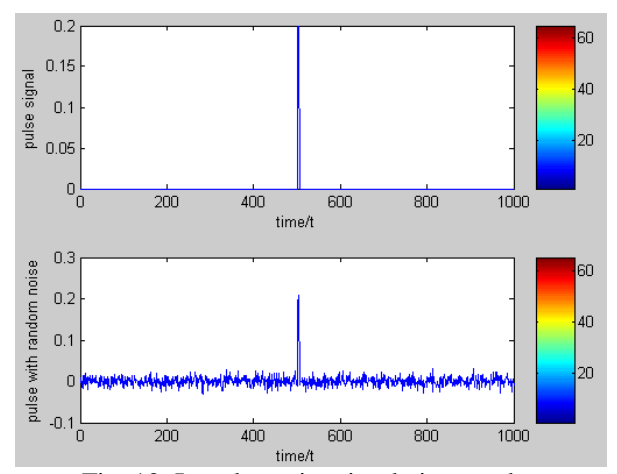

Fig. 10. Impulse noise simulation results.

In (3), where $A_{i}(t)$ is the pulse amplitude; $f_{i}$ is the pulse frequency, and the amplitude and frequency can be analyzed from the measurement data. $\varphi_{i}$ is the initial phase of the pulse which is a random number.

The production of narrowband noise in MATLAB is shown in Fig. 9.

Impulse noise can be simulated with a series of unit pulse amplitude and unit pulse width, as following equation:

$$
n_{\text {imp }}=\sum_{i=1}^{n} A_{i} i m p\left[\frac{t-t_{\alpha}, i}{t_{w}, i}\right]
$$

In (4), $\mathrm{A}_{\mathrm{i}}$ represents the pulse amplitude; $t_{\alpha}$ represents the pulse time interval; $t_{w}$ represents the pulse width, which is given by a large number of measurement results, and $i$ is a random variable. Select the appropriate coefficient, and the simulation result of equation (4) is shown in Fig. 10.

\section{LOW VOlTage POWER Line CHANNEL CHARACTERISTIC MODEL}

The power line model is an equivalent simulation model that can simulate the power line attenuation characteristics, impedance characteristics and noise characteristics. The more power line channel models currently used are multipath transmission models, as shown in Fig. 11.

There are many nodes in power line grids. As shown in Fig. 11, the signal transmits through a node B from point A to point D. Therefore, there are many possibilities for the path that the signal can transmit from $A$ to $D$, such as A-B-D, A-B-C-B-D, A-B-C-B-C-B-D, etc. That is, the signal may be reflected one or more times at the $\mathrm{BC}$ port, as the signal reaching the $\mathrm{D}$ point is the superimposed of the results after 0 or $\mathrm{N}$ reflections at the $\mathrm{BC}$ port, and there is a certain difference in phase and amplitude. To facilitate computational simulation, the simplified two-path transmission model is shown in Fig. 12.

The model mainly considers the attenuation and noise interference of the power line two-path transmission. The multiplicative delay interference and additive noise interference can be clearly seen from this model. The simulation results of the model can reflect the law of change of the measured data very well, so it can be concluded that the two-path model can reflect the law of change of the power line channel characteristics under normal conditions.
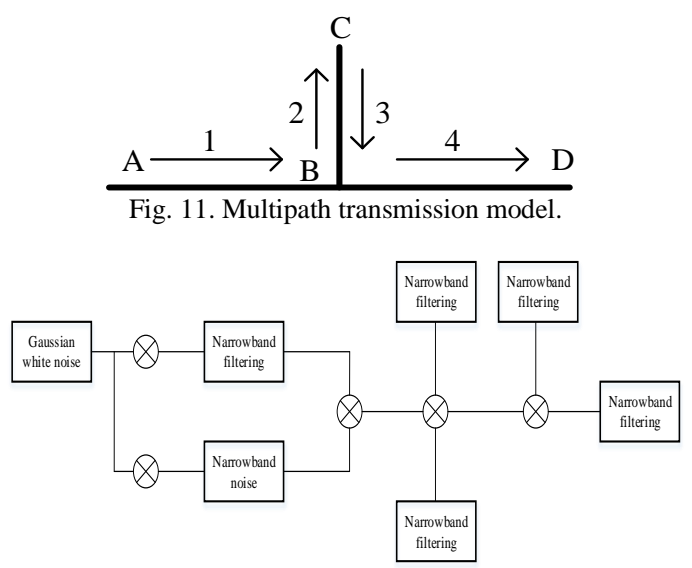

Fig. 12. Power line equivalent model.

\section{CONCLUSION}

Based on the measured data analyzing and theoretical research, the paper proposes a power line equivalent model, which can well reflect the power line channel characteristics in the laboratory environment and has certain practicability. The measured data and simulation results both show that the noise interference in the $100 \mathrm{kHz}$ to $500 \mathrm{kHz}$ frequency range 
is small and relatively stable, which is suitable for a frequency band of carrier communication. And the input impedance increases in the frequency range of $100 \mathrm{kHz}$ to $500 \mathrm{kHz}$ as the carrier frequency increases, so $100 \mathrm{kHz}$ to $150 \mathrm{kHz}$ is an ideal carrier signal frequency. This frequency has a large difference from the power frequency of the grid, so it's a better communication method which can be applied in commercial power.

\section{ACKNOWLEDGMENT}

The authors wish to thank our teacher Xixiu $\mathrm{Wu}$, who is the corresponding author. Her professional working experience and knowledge provided huge assistance on this researching. This work was supported in part by a grant from Chaoqun $\mathrm{Li}$. He provided the main equipment, and the place of experiment he choosed ensures the experiment done successfully. Also, the alma mater gives the studying opportunity and creating the chance meeting the scholars above. What's more, in the next research work, a smart communication solution can be proposed for smart home based on smart meter power line carrier communication.

\section{REFERENCES}

[1] Y. Zhang, S. Cheng, H. Cheng, and J. Nguimbis, "Modeling research in high frequency carrrier communication channel of low voltage power line," Automation of Electric Power Systems, no. 23, pp. 62-66, 2002.

[2] X. Geng and Y. Li, "Channel characteristics analysis and model research of low voltage power line communication," Power System Communication, vol. 4, no. 19, pp. 21-24, 2004.

[3] W. Cai, J. Le, C. Jin, C. Huang, and X. Zheng, "Survey of power line carrier communication channel modeling technology," Chinese Scientific Papers Online, vol. 6, no. 8, pp. 622-628, 2011

[4] J. Mitra and L. Lampe, "Coded narrowband transmission over noisy powerline channels," in Proc. IEEE International Symposium on Power Line Communications and Its Applications, ISPLC 2009, 2009.

[5] H. Wang, X. Wang, and Q. Hu, "Simulation of OFDM synchronization algorithm based on power line channel," Power System Communication, vol. 19, no. 19, pp. 26-31, 2008.

[6] Y. Dai, "Analysis of power line communication channel characteristics based on multi-conductor transmission line," Power System Communication, vol. 32, no. 12, pp. 59-63, 2011.
[7] S. Huttinger, J. Rupp, G. Griepentrog, P. Karols, and K. Dostert, "Derivation of statistical properties for mass transit power supply networks as powerline communication channel," in Proc. International Symposium on Power Line Communications and its Applications, 2005.

[8] S. Naiman, M. M. Kissaka, and N. H. Mvungi, "Energy meter reading and tampering protection through powerline communication channel," in Proc. 7th AFRICON Conf. in Africa, AFRICON, 2004.

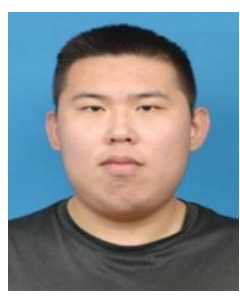

Bochuan Wang was born in Liaoning Province, China, in 1998. He is an undergraduate studying in WuHan University of Technology, WuHan, China. His profession is electrical engineering. He has worked on two programs: high voltage transmission line induction power and ultraviolet imaging device by corona discharge detection. He is interested in hardware development and High voltage technology.

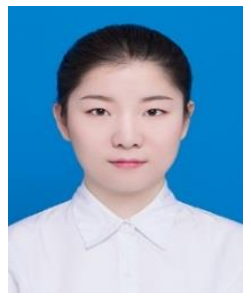

Zhuqing Xia was born in Hubei Province, China, in 1996. She received the B.E. degree in electrical engineering from Wuhan University of Technology, Wuhan, China. At current, she is a candidate of the M.E. degree in high voltage and insulation technology. At present, her profession is electrical engineering. Her research interests include GIS and switchgear.

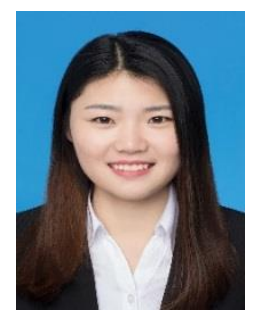

Xin Yang was born in Hubei Province, China, in 1996. She is now a candidate of the M.E. degree in high voltage and insulation technology. And her research interests include high voltage technology and electromagnetic compatibility.

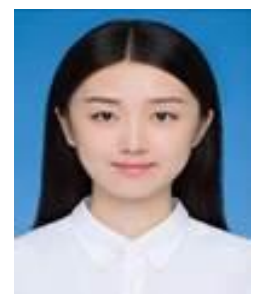

Zhining Yang was born in Liaoning Province, China, in 1994. She received the B.E. degree in electrical engineering from Wuhan University of Technology, Wuhan, China. At current, she is a candidate of the M.E. degree in high voltage and insulation technology. She is very interested in distribution line, especially lightning protect and grounding. 